\title{
Response to letter to the editor, regarding Evidence-based CPGs for the Periodic Eye Examination in Children Aged 0-5 Years in
}

\section{Canada}

Feb $5^{\text {th }} 2020$

To Prof. McCullough and collaborators,

Many thanks for the interest taken in these guidelines, and for taking the time to voice your reflections.

We are grateful for you to highlight how our paper might be misconstrued. The intent of the Committee was to point out the importance of complete eye examinations in all healthy children and at an early age. Please note the manuscript does not state a lower age limit for the comprehensive eye examination - only an upper age limit. There should be no question that children with obvious problems and risk factors require an eye examination.

We would like to emphasize the unique character of this multidisciplinary committee, which gave rise to the first set of guidelines on frequency of comprehensive examinations endorsed by more than one professional body. Indeed, the Canadian reality is that there is a diversity of health care providers caring for our children and, as stated in the paper "inequities in human, financial and healthcare resources in different regions of the country." As such, it was deemed critical to involve all the relevant professional bodies in this project, in order to foster interprofessional agreement, which carries more weight for public health and advocacy efforts. While working with multiple health professions may be challenging, we did, as a committee, agree for the first time in Canada and in publication that all children need a full eye examination at a young age. Once in the system, the frequency of eye examinations should be determined by the eye care professional.

When working intraprofessionally, it is much easier to deal with lack of evidence by using the consensus process. Consensus is much more of a challenge when working interprofessionally. In this case, we decided as a committee to look for what evidence was available and to specifically address amblyopia. This may have limited the scope of our paper but we are happy with the outcome.

Furthermore, it is important to clarify that the Committee did not state that primary health care providers "manage vision assessment by screening." As a multidisciplinary group, we did not exclude any practitioner's current procedures. We did however highlight the importance of complete eye examinations by eye care professionals and stated these were possible from birth.

Thank you for pointing us to the APHA and AOA guidelines which were not included in the review. While this was an oversight, their inclusion in the review are unlikely to have steered the outcome in a significantly different way. Indeed, as a multidisciplinary committee, we decided not to build on existing guidelines, where the content derived from consensus may be driven by a given, and dominant profession. Rather, in the context of the multidisciplinary approach, we agreed to conduct a novel review of the literature, relying on recognized methods, in order to obtain a set of guidelines applicable to the Canadian setting.

Respectfully,

Barbara E. Robinson, OD, MPH, PhD, FAAO

Professor Emeritus

School of Optometry and Vision Science

University of Waterloo

Benoit Tousignant, OD, MSc MPH, FAAO

Assistant Professor

School of Optometry

Université de Montréal 